\title{
Quality of Life in Children with Epilepsy in Private and Public Tertiary Care Centers in India
}

Purva Keni Karnavat ${ }^{1} \quad$ Anaita Udwadia Hegde ${ }^{1} \quad$ Shilpa Kulkarni ${ }^{2}$

\begin{abstract}
Address for correspondence Purva Keni Karnavat, DNB Pediatrics, Department of Pediatric Neurology, Jaslok Hospital and Research Centre, 9th floor, Pediatric Research Room, Mumbai, Maharashtra, India (e-mail: purvak121@gmail.com).
\end{abstract}

Int J Epilepsy 2018;5:28-37

\begin{abstract}
Keywords

- quality of life

- adverse effects

- pediatric epilepsy

- private and public

tertiary care centers

Background Pediatric epilepsy is associated with various comorbidities. It is known that children with epilepsy have a compromised health-related quality of life (QOL) and may be affected across physical, psychological, social, and educational domains.

Aims and Objectives (1) To study QOL in children with epilepsy from private and public outpatient departments, (2) study various risk factors affecting QOL, and (3) compare risk factors between groups.

Methods Sample consisted of 400 and 201 children aged 5 to 18 years from private and public settings with epilepsy taking antiepileptic drugs (AEDs) for at least 6 months. Structured case record form was used to collect data on sociodemographic, clinical, and treatment parameters. QOL was measured by the Pediatric Quality of Life Inventory (PedsQL) questionnaire (in the local language). Adverse effects were monitored by Pediatric Epilepsy Side Effects Questionnaire (PESQ). Univariate regression analysis was performed on the data $(p<0.005)$.

Results Overall PedsQL score was $67.21 \pm 29.391$ and $78.66 \pm 9.161$ in private and public settings, respectively $(p=0.000)$. QOL was affected significantly by age of the child, educational and economic background of the caregivers, age at the onset of epilepsy, seizure frequency, duration of treatment, number of drugs, presence of comorbidities, intelligence quotient (IQ) levels of children, and PESQ score in both the groups. Total PESQ score was $18.63 \pm 17.02$ and $7.69 \pm 10.646$ in private and public settings, respectively $(p=0.000)$.

Conclusions Children with epilepsy have a compromised QOL. Risk factors relating to QOL are numerous. Educational and economic status of caregivers plays a significant role in awareness of the problem.
\end{abstract}

\section{Introduction}

Epilepsy in children is frequently associated with ${ }^{1}$ comorbid conditions such as attention-deficit/hyperactivity disorder, autism, developmental disabilities, accidental injury, migraine, and depression/anxiety. Understanding the interrelationships among comorbidities, epilepsy, and their treatments is essential for optimal management of pediatric patients. ${ }^{2}$

Over the past three decades, the impact of pediatric epilepsy and its comorbidities on the lives of children has been occasionally evaluated using patient-reported outcomes (PROs) such as the health-related quality-of-life (HRQOL) index. Factors affecting the quality of life (QOL) in pediatric epilepsy include the age of onset of epilepsy, severity of seizures, cognitive impairment prevailing in children suffering from epilepsy, worry/concerns of the family, and psychological impact on them. A recent review summarizing various studies has shown that antiepileptic drugs (AEDs) may also have a negative impact on the child's well-being and daily functioning. ${ }^{3}$
DOI https://doi.org/

$10.1055 / \mathrm{s}-0038-1660774$

ISSN 2213-6320.
License terms

(ㅇ)(1) $\circledast$ 
There is a lot of inconsistency in data regarding the AEDs and their effects in impacting the HRQOL index. This may be influenced by the characteristics of the sample population included, the methodology by which the HRQOL index and the adverse events related to the AEDs were assessed. ${ }^{4-14}$ Most of the previous studies included mainly adolescents with epilepsy, whereas young children as well as patients with intellectual disabilities or comorbidities were rarely included. Many of the studies have solely evaluated how the number of AEDs impacted the HRQOL scores but importantly did not take into account their adverse effects. Finally, none of the previous studies compared the QOL in children at private and public settings. It is important to understand the impact of the sociodemographic factors in determining the QOL in these special children.

\section{Aims and Objectives}

The aim of our study was to evaluate the QOL, its risk factors in children with epilepsy in both private and public tertiary centers, and influence of the adverse effects using the ratings provided by the parents. Our secondary objective was to determine whether there is any difference in QOL rating and its determinants in both these settings.

\section{Materials and Methods}

\section{Participants}

The study was conducted over 12 months (August 2015 to August 2016) at the Pediatric Neurology Outpatient Department of two tertiary care units catering to different socioeconomic strata, henceforth referred to as "private" and "public" setting. Institutional ethical review board approval was obtained. The data were drawn from 601 (400 from private setting and 201 from public setting) children and adolescents with epilepsy (hereinafter being referred to as "children") and at least one parent who participated as a proxy rater.

All participants were assessed during their regular clinic visits (after an informed consent was obtained from caregivers prior to the study). Patients included in the study were in the age range 5 to 18 years, with or without active epilepsy (history of at least one seizure during the past year despite the constant use of AEDs) who were treated with AEDs for at least 6 months. Pediatric patients with severe neurological impairment or severe intellectual disability confounding QOL assessment were not considered. The intellectual abilities were assessed prior to the initiation of the study using Binet-Kamat test for intelligence. A structured case record form was used to collect data on sociodemographic, clinical, and treatment parameters.

\section{Questionnaire}

The Pediatric Quality of Life Inventory (PedsQL) was used for proxy rating of the QOL index..$^{15}$ Parents completed the prevalidated English, Hindi, Marathi, and Gujarati versions as per their language preference. ${ }^{15}$ The PedsQL instrument has 23 items allocated into four scales: physical ( 8 items), emotional (5 items), social (5 items), and school (5 items). The physical functioning score is computed by dividing the sum of the total items by the number of items answered in the physical scale (8 items). The psychosocial functioning score was calculated by computing the mean as the sum of the items divided by the number of items answered in the emotional, social, and school scales ( 15 items). Finally, the PedsQL total score was computed as the sum of all items divided by the number of items answered. Higher scores indicate better levels of functioning.

The adverse event profile was assessed using the Pediatric Epilepsy Side Effects Questionnaire (PESQ). ${ }^{16}$ It is a 19 -item instrument that assesses the presence and severity of the most frequent AED-related adverse effects noted in the preceding month. It is divided into five subgroups: cognitive (6 items), motor (4 items), behavior (3 items), general neurology (4 items), and weight ( 2 items). The severity of each symptom is rated on a 6 -point response scale $(1=$ not present to $6=$ high severity). Scaled scores are obtained for each domain as well as for total PESQ by using the equations found for each scale. Lower scores indicate lower severity of side-effects. Caregivers conversant in English completed the validated questionnaire whereas the remainder completed a locally validated questionnaire in their language of preference.

\section{Statistics}

Data were analyzed using univariate regression on SPSS version 20 . Descriptive data were expressed as mean \pm standard deviation (SD) and percentages. The PedsQL and PESQ scores were expressed as mean \pm SD with $95 \%$ confidence intervals $(\mathrm{CI})$. Categorical variables were compared using the chi-square test. Continuous variables were analyzed using the unpaired $t$-test and analysis of variance (parametric) and the Spearman correlation coefficient. Statistical significance was set at $p<0.05$.

\section{Results}

A. Demographic and clinical profile: The data on sociodemographic, clinical, and treatment parameters in both settings are depicted in - Table 1. It depicts all recorded variables that have a potential to influence the aspects of functioning. It shows a significant difference $(p<0.05)$ in the sociodemographic factors such as education of parents, annual income of family, sex, and intelligence quotient (IQ) of children when compared in both settings. Children in private setting had significantly more refractory epilepsies requiring more drugs and higher incidence of comorbidities as compared with their public counterparts.

B. PESQ scores in children with epilepsy: The total PESQ score was $18.63 \pm 17.02$ and $7.69 \pm 10.646$ in private and public settings, respectively, which shows that caregivers from public settings perceived fewer side effects in their children as compared with their private counterparts. The individual scaled scores seen in $\boldsymbol{- T a b l e} \mathbf{2}$ showed a similar finding on comparison.

C. PedsQL score in children with epilepsy: The overall PedsQL score was $67.21 \pm 29.391$ and $78.66 \pm 9.161$ in private and public settings, respectively, with a significant difference. The individual physical and psychosocial scores shown in - Table 3 had significantly higher values in the public setting. D. Relationship between QOL scores and clinical characteristics. 
Table 1 General characteristics of children $(n=601)$

\begin{tabular}{|c|c|c|c|}
\hline Characteristics & Private $(n=400)$ & Public $(n=201)$ & p-Value \\
\hline Age in years (Mean [SD]) & $11.210(4.36)$ & $10.035(3.32)$ & 0.001 \\
\hline Sex (male/female) (\%) & 243/157 (60.8/39.2) & $134 / 67(66.7 / 33.3)$ & 0.157 \\
\hline \multicolumn{4}{|l|}{ Father's education } \\
\hline Illiterate & $9(2.25 \%)$ & $8(4 \%)$ & \multirow[t]{5}{*}{0.000} \\
\hline School up to 10 th grade & $104(26 \%)$ & $114(56.7 \%)$ & \\
\hline Junior college & $67(16.75 \%)$ & $49(24.4 \%)$ & \\
\hline Graduation courses & $182(45.50 \%)$ & $27(13.4 \%)$ & \\
\hline Post graduation & $38(9.5 \%)$ & $3(1.5 \%)$ & \\
\hline \multicolumn{4}{|l|}{ Mother's education } \\
\hline Illiterate & $17(4.25 \%)$ & $20(10 \%)$ & \multirow[t]{5}{*}{0.000} \\
\hline School up to 10 th grade & $112(28 \%)$ & $135(67.2 \%)$ & \\
\hline Junior college & $71(17.75 \%)$ & $19(9.5 \%)$ & \\
\hline Graduation courses & $171(42.75 \%)$ & $24(11.9 \%)$ & \\
\hline Post graduation & $29(7.25 \%)$ & $3(1.5 \%)$ & \\
\hline Annual income of parents in rupees (lacs) (M [SD]) & $4.49(4.62)$ & $1.32(1.11)$ & 0.000 \\
\hline \multicolumn{4}{|l|}{ Type of epilepsy } \\
\hline Symptomatic & $244(61 \%)$ & $125(62.18 \%)$ & \multirow[t]{3}{*}{0.052} \\
\hline Idiopathic & $127(31.75 \%)$ & $71(35.32 \%)$ & \\
\hline Syndromic & $29(7.25 \%)$ & $5(2.5 \%)$ & \\
\hline \multicolumn{4}{|l|}{ Type of seizure } \\
\hline Generalized & $267(66.75 \%)$ & $94(46.76 \%)$ & \multirow[t]{2}{*}{0.000} \\
\hline Partial & $133(33.25 \%)$ & $107(53.23 \%)$ & \\
\hline Age at onset of epilepsy in years (M [SD]) & $3.58(3.885)$ & $4.05(3.570)$ & 0.150 \\
\hline Duration of epilepsy in years (M [SD]) & $6.82(4.542)$ & $5.33(3.512)$ & 0.000 \\
\hline \multicolumn{4}{|l|}{ Active epilepsy } \\
\hline Yes & $260(65 \%)$ & $119(59.2 \%)$ & \multirow[t]{2}{*}{0.251} \\
\hline No & $140(35 \%)$ & $82(40.8 \%)$ & \\
\hline \multicolumn{4}{|l|}{ Seizure frequency (\%) } \\
\hline 0 & $224(56 \%)$ & $131(65.2 \%)$ & \multirow[t]{3}{*}{0.094} \\
\hline 1 & $52(13 \%)$ & $22(10.9 \%)$ & \\
\hline$\geq 2$ & $124(31 \%)$ & $48(28.6 \%)$ & \\
\hline \multicolumn{4}{|l|}{ Duration of treatment in years (M [SD]) } \\
\hline$<1$ & $23(5.8 \%)$ & $21(10.4 \%)$ & \multirow[t]{3}{*}{0.033} \\
\hline $1-2$ & $32(8 \%)$ & $23(11.4 \%)$ & \\
\hline$>2$ & $345(86.2 \%)$ & $157(78.1 \%)$ & \\
\hline \multicolumn{4}{|l|}{ Number of AEDs } \\
\hline 1 & $185(46.2 \%)$ & $125(62.2 \%)$ & \multirow[t]{3}{*}{0.001} \\
\hline 2 & $116(29 \%)$ & $45(22.5 \%)$ & \\
\hline$\geq 3$ & $99(24.8 \%)$ & $31(15.4 \%)$ & \\
\hline \multicolumn{4}{|l|}{ Comorbidities } \\
\hline Yes & $306(76.5 \%)$ & $116(57.7 \%)$ & \multirow[t]{2}{*}{0.000} \\
\hline No & $94(23.5 \%)$ & $85(42.3 \%)$ & \\
\hline
\end{tabular}


Table 1 (Continued)

\begin{tabular}{|c|c|c|c|}
\hline Characteristics & Private $(n=400)$ & Public ( $n=201$ ) & $p$-Value \\
\hline \multicolumn{4}{|c|}{ Type of comorbidities } \\
\hline Motor & $33(8.25 \%)$ & $3(1.49 \%)$ & \multirow[t]{5}{*}{0.000} \\
\hline Cognitive & $51(12.75 \%)$ & $23(11.44 \%)$ & \\
\hline Behavior & $74(18.5 \%)$ & $22(10.94 \%)$ & \\
\hline Combined & $148(37 \%)$ & $68(33.83 \%)$ & \\
\hline Absent & $94(23.5 \%)$ & $85(42.3 \%)$ & \\
\hline \multicolumn{4}{|l|}{ IQ } \\
\hline$<70$ & $143(35.8 \%)$ & $49(24.4 \%)$ & \multirow[t]{4}{*}{0.026} \\
\hline $70-89$ & $106(26.5 \%)$ & $62(30.8 \%)$ & \\
\hline $90-110$ & $149(37.2 \%)$ & $90(44.8 \%)$ & \\
\hline$>110$ & $2(0.5 \%)$ & $0(0 \%)$ & \\
\hline
\end{tabular}

Abbreviations: AED, antiepileptic drug; IQ, intelligence quotient; SD, standard deviation.

Note: Values in bold are significant.

Table 2 PESQ scores in CWE

\begin{tabular}{|l|l|l|l|}
\hline Characteristics & Private $(\boldsymbol{n}=\mathbf{4 0 0})$ & Public $(\boldsymbol{n}=\mathbf{2 0 1})$ & $\boldsymbol{p}$-Value \\
\hline & M (SD) & M (SD) & \\
\hline Cognitive & $23.64(24.55)$ & $8.99(13.58)$ & $\mathbf{0 . 0 0 0}$ \\
\hline Motor & $13.80(21.18)$ & $3.53(10.96)$ & $\mathbf{0 . 0 0 0}$ \\
\hline Behavior & $21.74(24.21)$ & $11.2(16.84)$ & $\mathbf{0 . 0 0 0}$ \\
\hline General neurology & $14.25(19.01)$ & $6.08(9.83)$ & $\mathbf{0 . 0 0 0}$ \\
\hline Weight & $17.79(25.43)$ & $8.15(20.69)$ & $\mathbf{0 . 0 0 0}$ \\
\hline Total & $18.63(17.02)$ & $7.69(10.646)$ & $\mathbf{0 . 0 0 0}$ \\
\hline
\end{tabular}

Abbreviations: CWE, children with epilepsy; PESQ, Pediatric Epilepsy Side effect Questionnaire; SD, standard deviation.

Table 3 PedsQL score in CWE

\begin{tabular}{|l|l|l|l|}
\hline Characteristics & \multicolumn{1}{l|}{ Private $(\boldsymbol{n}=400)$} & Public $(\boldsymbol{n}=\mathbf{2 0 1})$ & $\boldsymbol{p}$-Value \\
\hline \multicolumn{7}{|c|}{ M (SD) } & $\mathbf{0 . 0 0 0}$ \\
\hline Physical & $67.86(27.601)$ & $82.82(22.716)$ & $\mathbf{0 . 0 0 0}$ \\
\hline Psychosocial & $66.05(20.371)$ & $74.56(19.458)$ & $\mathbf{0 . 0 0 0}$ \\
\hline Overall & $67.21(29.391)$ & $78.66(19.161)$ & \\
\hline
\end{tabular}

Abbreviations: CWE, children with epilepsy; PedsQL; Pediatric Quality of Life Inventory; SD, standard deviation.

\section{Private Setting}

- Table 4 shows the relationship of sociodemographic and clinical variables with the overall physical, psychosocial, and overall PedsQL score. The mean PedsQL score was significantly higher in patients with partial seizures, onset of epilepsy at a later age, lower seizure frequency, lower duration of epilepsy, lower number of drugs, absence of comorbidities, and IQ greater than 90. Among children with comorbidities, those with motor and combined comorbidities scored significantly lower. Mean PedsQL scores were higher in lesser duration of epilepsy though not significant.

\section{Public Setting}

- Table 5 shows the relationship of sociodemographic and clinical variables with the overall physical, psychosocial, and overall PedsQL score. The mean PedsQL scores were significantly higher in children with low seizure frequency, lower duration of treatment, lesser number of drugs, absence of comorbidities, and higher IQs.

\section{Discussion}

\section{PedsQL in Both Settings}

The overall PedsQL was 67.21 and 78.66 in private and public setting, respectively, which was comparable to a similar study done in Serbia using the same scale. ${ }^{17}$ It was noted that across both the settings in our study, psychosocial scores were lower than the physical scores. Jovanovic et al ${ }^{17}$ too noted that AEDs played a huge impact on the psychosocial functioning of children in their study. It can be thus 
Table 4 PedsQL score and clinical characteristics in private setting

\begin{tabular}{|c|c|c|c|c|c|c|}
\hline \multirow[t]{2}{*}{ Characteristics } & \multicolumn{2}{|c|}{ Physical PedsQL score } & \multicolumn{2}{|c|}{ Psychosocial PedsQL score } & \multicolumn{2}{|c|}{ Overall PEDsQL score } \\
\hline & Mean \pm SD & $p$-Value & Mean \pm SD & $p$-Value & Mean \pm SD & $p$-Value \\
\hline \multicolumn{7}{|l|}{ Age in years } \\
\hline $5-10$ & $64.09 \pm 28.72$ & & $64.13 \pm 21.02$ & & $104.23 \pm 565.32$ & \\
\hline$>10-15$ & $70.19 \pm 27.50$ & & $67.68 \pm 18.49$ & & $68.99 \pm 20.23$ & \\
\hline$>15$ & $74.02 \pm 23.21$ & $p 0.014$ & $68.45 \pm 21.26$ & $p 0.162$ & $71.55 \pm 19.77$ & $p 0.694$ \\
\hline \multicolumn{7}{|l|}{ Sex } \\
\hline Male & $67.77 \pm 27.94$ & & $66.29 \pm 19.99$ & & $67.45 \pm 24.15$ & \\
\hline Female & $68.0 \pm 27.15$ & $p 0.937$ & $65.66 \pm 20.99$ & $p 0.763$ & $117.75 \pm 639.34$ & $p 0.22$ \\
\hline \multicolumn{7}{|l|}{ Father's education } \\
\hline Illiterate & $64.55 \pm 33.81$ & & $58.14 \pm 24.31$ & & $60.78 \pm 27.98$ & \\
\hline School up to 10 th grade & $69.16 \pm 27.27$ & & $67.35 \pm 19.79$ & & $68.37 \pm 21.18$ & \\
\hline Junior college & $71.81 \pm 25.46$ & $p 0.357$ & $68.91 \pm 18.32$ & & $70.56 \pm 19.71$ & \\
\hline Graduation courses & $65.02 \pm 27.97$ & & $64.77 \pm 20.54$ & & $109.18 \pm 593.97$ & \\
\hline Post graduation & $71.69 \pm 28.65$ & & $65.48 \pm 23.50$ & $p 0.429$ & $68.95 \pm 21.86$ & $p 0.91$ \\
\hline \multicolumn{7}{|l|}{ Mother's education } \\
\hline Illiterate & $69.43 \pm 31.52$ & & $70.18 \pm 24.32$ & & $69.76 \pm 26.64$ & \\
\hline School up to 10 th grade & $69.44 \pm 26.29$ & & $66.29 \pm 20.18$ & & $68.32 \pm 20.53$ & \\
\hline Junior college & $66.21 \pm 28.52$ & $p 0.942$ & $65.78 \pm 19.14$ & & $66.39 \pm 21.08$ & \\
\hline Graduation courses & $67.23 \pm 28.00$ & & $65.19 \pm 20.21$ & & $66.13 \pm 21.76$ & \\
\hline Post graduation & $68.52 \pm 27.04$ & & $68.33 \pm 23.33$ & $p 0.846$ & $68.42 \pm 25.58$ & $p 0.0108$ \\
\hline $\begin{array}{l}\text { Annual income of parents in } \\
\text { rupees (lacs) }\end{array}$ & $67.86 \pm 37.60$ & $p<0.00001$ & $66.05 \pm 20.37$ & $p<0.00001$ & $67.21 \pm 21.39$ & $p<0.00001$ \\
\hline \multicolumn{7}{|l|}{ Type of epilepsy } \\
\hline Symptomatic & $68.09 \pm 27.71$ & & $63.98 \pm 19.59$ & & $64.18 \pm 21.08$ & \\
\hline Idiopathic & $68.53 \pm 27.94$ & $p=0.6023$ & $70.54 \pm 21.66$ & & $74.05 \pm 20.77$ & \\
\hline Syndromic & $62.93 \pm 25.54$ & & $63.76 \pm 18.33$ & $p=0.010$ & $62.74 \pm 20.21$ & $p 0.000$ \\
\hline \multicolumn{7}{|l|}{ Type of seizure } \\
\hline Generalized & $67.2 \pm 27.31$ & & $64.27 \pm 20.07$ & & $65.28 \pm 21.62$ & \\
\hline Partial & $69.1 \pm 28.22$ & $p=0.528$ & $69.62 \pm 20.56$ & $p=0.013$ & $71.09 \pm 20.45$ & $p 0.010$ \\
\hline \multicolumn{7}{|c|}{ Age at onset of epilepsy in years } \\
\hline $0-5$ & $66.36 \pm 28.29$ & & $63.91 \pm 20.13$ & & $64.29 \pm 21.37$ & \\
\hline$>5-10$ & $73.27 \pm 24.06$ & & $70.1 \pm 20.45$ & & $73.99 \pm 19.78$ & \\
\hline$>10-15$ & $64.27 \pm 29.19$ & $p=0.114$ & $78.57 \pm 17.36$ & & $50.44 \pm 17.72$ & \\
\hline$>15$ & $83.75 \pm 23.22$ & & $76.97 \pm 17.25$ & $p=0.001$ & $78.25 \pm 16.08$ & $p 0.00004$ \\
\hline \multicolumn{7}{|l|}{ Duration of epilepsy in years } \\
\hline$\geq 2$ & $72 \pm 26.12$ & & $70.98 \pm 21.69$ & & $71.69 \pm 23.30$ & \\
\hline $2-5$ & $70.04 \pm 26.63$ & & $65.95 \pm 20.88$ & & $67.58 \pm 21.98$ & \\
\hline$>5-10$ & $62.85 \pm 28.18$ & $p=0.033$ & $64.96 \pm 20.01$ & & $65.96 \pm 21.34$ & \\
\hline$>10$ & $71.35 \pm 27.84$ & & $63.59 \pm 18.74$ & $p=0.108$ & $65 \pm 18.50$ & $p 0.191$ \\
\hline \multicolumn{7}{|l|}{ Active epilepsy } \\
\hline Yes & $67.36 \pm 29.48$ & & $65.82 \pm 20.8$ & & $97.67 \pm 497$ & \\
\hline No & $68.8 \pm 23.78$ & $p 0.62$ & $66.47 \pm 19.62$ & $\mathrm{p} 0.762$ & $67.73 \pm 19.76$ & $p 0.476$ \\
\hline
\end{tabular}


Table 4 (Continued)

\begin{tabular}{|c|c|c|c|c|c|c|}
\hline \multirow[t]{2}{*}{ Characteristics } & \multicolumn{2}{|c|}{ Physical PedsQL score } & \multicolumn{2}{|c|}{ Psychosocial PedsQL score } & \multicolumn{2}{|c|}{ Overall PEDsQL score } \\
\hline & Mean \pm SD & $p$-Value & Mean \pm SD & $p$-Value & Mean \pm SD & $p$-Value \\
\hline \multicolumn{7}{|l|}{ Seizure frequency } \\
\hline 0 & $69.93 \pm 27.16$ & & $69.17 \pm 20.29$ & & $69.99 \pm 21.81$ & \\
\hline 1 & $67.30 \pm 26.55$ & $p 0.194$ & $65.399 \pm 18.77$ & & $69.46 \pm 17.74$ & \\
\hline$\geq 2$ & $64.35 \pm 28.65$ & & $60.67 \pm 20.16$ & $p 0.0008$ & $61.25 \pm 20.95$ & $p 0.0008$ \\
\hline \multicolumn{7}{|c|}{ Duration of treatment in years } \\
\hline$<1$ & $58.93 \pm 20.91$ & & $61.42 \pm 18.79$ & & $62.52 \pm 18.56$ & \\
\hline $1-2$ & $71.44 \pm 18.52$ & $p 0.226$ & $69.31 \pm 17.06$ & & $71.08 \pm 17.42$ & \\
\hline$>2$ & $68.12 \pm 28.60$ & & $66.0 \pm 20.74$ & $p 0.367$ & $67.16 \pm 21.87$ & $p 0.341$ \\
\hline \multicolumn{7}{|l|}{ Number of AEDs } \\
\hline 1 & $67.97 \pm 28.17$ & & $70.41 \pm 20.21$ & & $72.60 \pm 21.24$ & \\
\hline 2 & $68.64 \pm 27.15$ & $p 0.913$ & $64.95 \pm 21.22$ & & $66.12 \pm 21.47$ & \\
\hline$\geq 3$ & $67.04 \pm 27.05$ & & $59.17 \pm 17.6$ & $p 0.000$ & $58.41 \pm 18.44$ & $p<0.00001$ \\
\hline \multicolumn{7}{|l|}{ Comorbidities } \\
\hline Yes & $67.34 \pm 27.44$ & & $62.21 \pm 18.66$ & & $62.98 \pm 20.04$ & \\
\hline No & $69.9 \pm 27.92$ & $p 0.431$ & $78.54 \pm 20.78$ & $p<0.00001$ & $81 \pm 19.86$ & $p<0.00001$ \\
\hline \multicolumn{7}{|c|}{ Type of comorbidities } \\
\hline Motor & $63.56 \pm 25.09$ & & $59.975 \pm 20.57$ & & $58.89 \pm 21.67$ & \\
\hline Cognitive & $69.43 \pm 27.23$ & & $64.56 \pm 17.83$ & & $67.45 \pm 18.74$ & \\
\hline Behavior & $67.43 \pm 28.66$ & $p 0.824$ & $67.31 \pm 18.74$ & & $70.08 \pm 19.01$ & \\
\hline Combined & $67.07 \pm 27.94$ & & $58.54 \pm 18.19$ & $p=0.006$ & $58.32 \pm 19.23$ & p 0.00007 \\
\hline \multicolumn{7}{|l|}{ IQ } \\
\hline$<70$ & $66.55 \pm 27.05$ & & $58.58 \pm 18.81$ & & $56.97 \pm 20.43$ & \\
\hline $70-89$ & $66.76 \pm 30.7$ & & $63.16 \pm 17.33$ & & $65.6 \pm 17.72$ & \\
\hline $90-110$ & $69.97 \pm 25.78$ & $p 0.653$ & $75.20 \pm 20.53$ & & $78.08 \pm 19.67$ & \\
\hline$>110$ & $78.12 \pm 13.26$ & & $70.83 \pm 3.53$ & $p<0.00001$ & $75.78 \pm 3.75$ & $p<0.00001$ \\
\hline \multicolumn{7}{|l|}{ PESQ } \\
\hline Cognitive & & $p<0.00001$ & & $p<0.00001$ & & $p<0.00001$ \\
\hline Motor & & $p<0.00001$ & & $p<0.00001$ & & $p<0.00001$ \\
\hline Behavior & & $p<0.00001$ & & $p<0.00001$ & & $p<0.00001$ \\
\hline General neurology & & $p<0.00001$ & & $p<0.00001$ & & $p<0.00001$ \\
\hline Weight & & $p<0.00001$ & & $p<0.00001$ & & $p<0.00001$ \\
\hline Total & & $p<0.00001$ & & $p<0.00001$ & & $p<0.00001$ \\
\hline
\end{tabular}

Abbreviations: IQ, intelligence quotient; PedsQL; Pediatric Quality of Life Inventory; PESQ, Pediatric Epilepsy Side Effects Questionnaire; SD, standard deviation.

concluded that most of the variables have a higher impact on psychosocial QOL scores rather than physical QOL. Our data showed that parents from private setting scored their children less on QOL when compared with their public counterparts. This may be because that parents coming to private setting have higher expectations from treatment when compared with public setting despite the similar access to treatment. It is important to note here that the datasets from public and private settings are unequal in terms of refractory epilepsy, number of AEDs and comorbidities. Recently, Modi et a ${ }^{18}$ demonstrated the PedsQL Epilepsy Module as a reliable measure of HRQOL with strong evidence of its validity across the epilepsy spectrum in both clinical and research settings. 
Table 5 PedsQL score and clinical characteristics in public setting

\begin{tabular}{|c|c|c|c|c|c|c|}
\hline \multirow[t]{2}{*}{ Characteristics } & \multicolumn{2}{|c|}{ Physical PedsQL score } & \multicolumn{2}{|c|}{ Psychosocial PedsQL score } & \multicolumn{2}{|c|}{ Overall PedsQL score } \\
\hline & Mean \pm SD & $p$-Value & Mean \pm SD & $p$-Value & Mean \pm SD & $p$-Value \\
\hline \multicolumn{7}{|l|}{ Age in years } \\
\hline $5-10$ & $80.005 \pm 25.77$ & & $73.55 \pm 19.93$ & & $76.87 \pm 20.66$ & \\
\hline$>10-15$ & $87.136 \pm 17.53$ & $p 0.107$ & $76.25 \pm 18.87$ & & $81.53 \pm 17.13$ & \\
\hline$>15$ & $85.268 \pm 13.39$ & & $74.46 \pm 18.22$ & $p 0.659$ & $79.69 \pm 14.02$ & $p 0.271$ \\
\hline \multicolumn{7}{|l|}{ Sex } \\
\hline Male & $81.23 \pm 24.59$ & & $73.62 \pm 19.83$ & & $77.48 \pm 19.95$ & \\
\hline Female & $85.99 \pm 18.16$ & $p 0.162$ & $76.42 \pm 18.69$ & $p 0.334$ & $81.03 \pm 17.37$ & $p 0.217$ \\
\hline \multicolumn{7}{|l|}{ Father's education } \\
\hline Illiterate & $78.52 \pm 18.02$ & & $73.88 \pm 17.86$ & & $75.97 \pm 16.33$ & \\
\hline $\begin{array}{l}\text { School up to } \\
\text { 10th grade }\end{array}$ & $83.15 \pm 23.30$ & & $74.84 \pm 19.76$ & & $78.82 \pm 19.94$ & \\
\hline Junior college & $85.44 \pm 19.41$ & $p 0.616$ & $74.65 \pm 19.71$ & $p 0.996$ & $80.14 \pm 16.77$ & $p 0.913$ \\
\hline $\begin{array}{l}\text { Graduation } \\
\text { courses }\end{array}$ & $79.51 \pm 23.86$ & & $73.15 \pm 19.15$ & & $76.76 \pm 20.14$ & \\
\hline Post graduation & $68.75 \pm 51.44$ & & $75.55 \pm 19.31$ & & $72.99 \pm 33.28$ & \\
\hline \multicolumn{7}{|l|}{ Mother's education } \\
\hline Illiterate & $87.19 \pm 19.74$ & & $75.71 \pm 16.08$ & & $81.21 \pm 16.17$ & \\
\hline $\begin{array}{l}\text { School up to } \\
\text { 10th grade }\end{array}$ & $83.70 \pm 21.05$ & & $75.24 \pm 19.00$ & & $79.41 \pm 18.01$ & \\
\hline Junior college & $87.5 \pm 12.32$ & & $78.24 \pm 13.54$ & $p 0.297$ & $82.87 \pm 10.82$ & $p 0.019$ \\
\hline $\begin{array}{l}\text { Graduation } \\
\text { courses }\end{array}$ & $76.82 \pm 29.57$ & $p 0.0008$ & $68.5 \pm 325.47$ & & $72.88 \pm 26.24$ & \\
\hline Post graduation & $32.29 \pm 45.54$ & & $60 \pm 31.22$ & & $47.48 \pm 37.19$ & \\
\hline $\begin{array}{l}\text { Annual income of } \\
\text { parents in } \\
\text { rupees (lacs) }\end{array}$ & $82.82 \pm 22.72$ & $p<0.00001$ & $74.54 \pm 19.41$ & $p<0.00001$ & $78.66 \pm 19.19$ & $p<0.00001$ \\
\hline \multicolumn{7}{|l|}{ Type of epilepsy } \\
\hline Symptomatic & $80.95 \pm 24.11$ & & $72.81 \pm 17.83$ & & $76.99 \pm 18.55$ & \\
\hline Idiopathic & $86.47 \pm 19.17$ & $p 0.229$ & $77.9 \pm 21.64$ & & $81.96 \pm 19.63$ & \\
\hline Syndromic & $77.5 \pm 31.04$ & & $70.01 \pm 21.42$ & $p 0.183$ & $73.81 \pm 25.28$ & $p 0.185$ \\
\hline \multicolumn{7}{|l|}{ Type of seizure } \\
\hline Generalized & $83.29 \pm 21.55$ & & $74.44 \pm 20.55$ & & $78.98 \pm 19.11$ & \\
\hline Partial & $82.4 \pm 23.79$ & $p 0.779$ & $74.63 \pm 18.45$ & $p 0.947$ & $78.38 \pm 19.29$ & $p 0.827$ \\
\hline \multicolumn{7}{|c|}{ Age at onset of epilepsy in years } \\
\hline $0-5$ & $80.18 \pm 25.31$ & & $72.899 \pm 19.15$ & & $76.59 \pm 20.12$ & \\
\hline$>5-10$ & $87.33 \pm 16.47$ & & $77.664 \pm 20.01$ & & $82.33 \pm 17.35$ & \\
\hline$>10-15$ & $90.62 \pm 10.17$ & $p 0.067$ & $77.8 \pm 18.51$ & & $84.19 \pm 12.56$ & \\
\hline$>15$ & - & & - & $p 0.253$ & - & $p 0.101$ \\
\hline \multicolumn{7}{|c|}{ Duration of epilepsy in years } \\
\hline$\geq 2$ & $81.44 \pm 28.56$ & & $74.90 \pm 24.22$ & & $78.27 \pm 24.96$ & \\
\hline $2-5$ & $81.78 \pm 24.99$ & & $76.67 \pm 18.16$ & & $79.23 \pm 19.99$ & \\
\hline$>5-10$ & $83.36 \pm 17.96$ & $p 0.782$ & $72.58 \pm 18.61$ & & $77.89 \pm 15.63$ & \\
\hline$>10$ & $87.33 \pm 15.50$ & & $74.64 \pm 12.26$ & $p 0.684$ & $80.85 \pm 12.77$ & $p 0.932$ \\
\hline
\end{tabular}


Table 5 (Continued)

\begin{tabular}{|c|c|c|c|c|c|c|}
\hline \multirow[t]{2}{*}{ Characteristics } & \multicolumn{2}{|c|}{ Physical PedsQL score } & \multicolumn{2}{|c|}{ Psychosocial PedsQL score } & \multicolumn{2}{|c|}{ Overall PedsQL score } \\
\hline & Mean \pm SD & $p$-Value & Mean \pm SD & $p$-Value & Mean \pm SD & $p$-Value \\
\hline \multicolumn{7}{|l|}{ Active epilepsy } \\
\hline Yes & $83.23 \pm 20.58$ & & $73.84 \pm 17.86$ & & $78.46 \pm 17.36$ & \\
\hline No & $82.23 \pm 25.62$ & $p 0.76$ & $75.57 \pm 21.53$ & $p 0.536$ & $78.96 \pm 21.61$ & $p 0.854$ \\
\hline \multicolumn{7}{|c|}{ Seizure frequency } \\
\hline 0 & $85.69 \pm 20.79$ & & $77.17 \pm 18.67$ & & $81.44 \pm 17.85$ & \\
\hline 1 & $83.71 \pm 24.93$ & $p 0.014$ & $74.32 \pm 22.85$ & & $78.51 \pm 23.1$ & \\
\hline$\geq 2$ & $74.59 \pm 25.11$ & & $67.46 \pm 17.74$ & $p 0.012$ & $71.16 \pm 19.07$ & $p 0.006$ \\
\hline \multicolumn{7}{|c|}{ Duration of treatment in years } \\
\hline$<1$ & $95.09 \pm 8.06$ & & $88.45 \pm 11.44$ & & $91.70 \pm 9.14$ & \\
\hline $1-2$ & $83.29 \pm 21.54$ & $p 0.029$ & $76.08 \pm 24.25$ & & $79.68 \pm 22.11$ & \\
\hline$>2$ & $81.11 \pm 23.76$ & & $72.45 \pm 18.77$ & $p 0.001$ & $76.77 \pm 19.089$ & $p 0.003$ \\
\hline \multicolumn{7}{|l|}{ Number of AEDs } \\
\hline 1 & $85.48 \pm 22.54$ & & $78.28 \pm 18.89$ & & $81.89 \pm 19.1$ & \\
\hline 2 & $83.09 \pm 17.02$ & $p 0.009$ & $68.67 \pm 18.23$ & & $75.92 \pm 15.14$ & \\
\hline$\geq 3$ & $71.69 \pm 27.52$ & & $67.97 \pm 19.86$ & $p 0.002$ & $69.61 \pm 21.54$ & $p 0.002$ \\
\hline \multicolumn{7}{|l|}{ Comorbidities } \\
\hline Yes & $79.45 \pm 24.07$ & & $68.83 \pm 16.95$ & & $74.16 \pm 17.80$ & \\
\hline No & $87.41 \pm 19.97$ & $p 0.013$ & $82.33 \pm 19.93$ & $p<0.00001$ & $84.81 \pm 19.34$ & $p 0.00008$ \\
\hline \multicolumn{7}{|c|}{ Type of comorbidities } \\
\hline Motor & $79.17 \pm 11.83$ & & $73.33 \pm 18.03$ & & $76.24 \pm 13.51$ & \\
\hline Cognitive & $85.21 \pm 22.39$ & & $74.12 \pm 17.24$ & & $78.84 \pm 18.16$ & \\
\hline Behavior & $84.75 \pm 17.88$ & $p 0.822$ & $76.80 \pm 15.71$ & & $80.97 \pm 15.8$ & \\
\hline Combined & $80.81 \pm 25.64$ & & $74.09 \pm 19.57$ & $p 0.941$ & $77.46 \pm 20.48$ & $p 0.894$ \\
\hline \multicolumn{7}{|l|}{ IQ } \\
\hline$<70$ & $74.6 \pm 25.36$ & & $65.9 \pm 14.59$ & & $69.96 \pm 17.25$ & \\
\hline $70-89$ & $82.22 \pm 24.07$ & & $71.86 \pm 18.88$ & & $77.30 \pm 18.93$ & \\
\hline $90-110$ & $87.71 \pm 18.82$ & $p 0.004$ & $81.1 \pm 19.95$ & & $84.33 \pm 18.52$ & \\
\hline$>110$ & - & & - & p 0.00002 & - & $p 0.00008$ \\
\hline \multicolumn{7}{|l|}{ PESQ } \\
\hline Cognitive & & $p<0.00001$ & & $p<0.00001$ & & $p<0.00001$ \\
\hline Motor & & $p<0.00001$ & & $p<0.00001$ & & $p<0.00001$ \\
\hline Behavior & & $p<0.00001$ & & $p<0.00001$ & & $p<0.00001$ \\
\hline $\begin{array}{l}\text { General } \\
\text { neurology }\end{array}$ & & $p<0.00001$ & & $p<0.00001$ & & $p<0.00001$ \\
\hline Weight & & $p<0.00001$ & & $p<0.00001$ & & $p<0.00001$ \\
\hline Total & & $p<0.00001$ & & $p<0.00001$ & & $p<0.00001$ \\
\hline
\end{tabular}

Abbreviations: IQ, intelligence quotient; PedsQL; Pediatric Quality of Life Inventory; PESQ, Pediatric Epilepsy Side effect Questionnaire; SD, standard deviation.

\section{Sociodemographic Variables and PedsQL}

The study population from private setting had parents with higher educational background and higher income than their public counterparts. Educational status of the mother had a significant bearing over the QOL in these children in both the groups. Between both the groups, higher educational status, particularly of mothers, recorded lower QOL scores. Maternal education explained significant amount of variance in QOL scores of children in previous studies too. ${ }^{19,20}$ However, in both these studies higher income and 
higher education contributed to better scores of QOL. Children in whom onset of epilepsy was at a younger age had lower QOL scores in comparison to increasing ages in both settings. Furthermore, these children had higher rate of comorbidities in both groups in our study population. The onset of seizures at a lower age in adults was associated with a poor QOL in earlier studies; though they could not give significance. ${ }^{21,22}$ Children with higher IQs in both settings seemed to have a better QOL due to better perception and could take care of themselves more effectively. In addition, in our study population many of children with better IQs were also associated with lesser comorbidities and better seizure control; all these variables add to QOL. A recently published Canadian study showed that emotional wellbeing of families had an impact on QOL in children with epilepsies, ${ }^{23}$ thus suggesting that clinicians should adopt family-centered treatment approach.

\section{Epilepsy Variables and PedsQL}

Our study concluded lower seizure frequency, lesser number of drugs, and absence of comorbidities in children with better QOL scores in both settings. Earlier studies have shown a direct correlation between increasing severity of epilepsy and lower QOL scores. ${ }^{20,24,25}$ Our study confirms that polytherapy is associated with poor QOL as shown in literature. A systematic review of studies on pediatric epilepsy published in 2011 showed that it has a significant influence on the HRQOL issues, particularly across the physical, psychological, social, and school-related domains. ${ }^{3}$ Age at epilepsy onset, number of AEDs, and parental depression were important HRQOL predictors, but specific to adolescents only, seizure worry/concerns and side effects of AEDs were found as strong predictors. ${ }^{3}$

\section{Adverse-Effect Profile (PESQ) and PedsQL}

Adverse effects are frequently reported in pediatric epilepsy. The higher the number of AEDs taken, the higher the occurrence of adverse effects. ${ }^{26}$ Our data suggested a higher likelihood of adverse effects in private setting than public setting, which could be due to the skewed dataset. Children in private setting had more severe epilepsies and higher rate of comorbidities. However, in both groups, behavior and cognitive side effects were perceived more than physical side effects. Previous studies have demonstrated that AEDs have a significant bearing on cognition and behavior profile of children. ${ }^{17,19,20,24}$ Evaluating the impact of AED-related adverse effects on the HRQOL scale is extremely challenging as all symptoms covered by a questionnaire are not necessarily due to the adverse effects of AEDs (such as a poor concentration or memory problems) but may in fact be genuine neuropsychological or neuropsychiatric problems prevalent due to the presence of epilepsy. ${ }^{27}$

\section{Strengths}

The study sample size is large in number when compared with previous studies. We have included children with low IQ scores, who were excluded in the previous studies. The questionnaires (PESQ and PedsQL) were given to caregivers in the best understood language thereby reducing the bias due to linguistic reasons.

\section{Limitations}

There is a sample size incongruence (private: 401 and public: 201) in our dataset. The study does not include children with severe neurological impairment or intellectual disability who also experience the adverse effects and thereby compromise their QOL.

\section{Conclusions}

Quality of life in children with epilepsy is affected across all domains irrespective of the setup. Educational status of parents, specifically of the mother, is an important contributor toward QOL. Better financial status implied better amenities and better physical scores; however, it also gave lower psychosocial scores probably due to higher expectation from the caregivers and epilepsy-related variables that were observed. Other factors such as frequency of seizures, number of drugs, presence and type of comorbidities, and IQ of the child contributed significantly toward QOL. Efficacy of the drugs seemed to be a primary goal in both setups; tolerability of the drugs was an important factor in private setup. Side effects of the drugs played a major role in determining QOL, and hence noting side effects at every consult can be useful.

\section{Funding \\ None.}

\section{Conflict of Interests}

None.

\section{Acknowledgments}

We would like to acknowledge the support of Mr. Dharmendra P. Singh in helping with the statistical analysis and Miss Sarita Londhe for administrative work. We would like to thank J. Varni and D. A. Morita for letting us use PedsQL and PESQ scales.

\section{References}

1 Camfield P, Camfield C. Incidence, prevalence and aetiology of seizures and epilepsy in children. Epileptic Disord 2015;17(2):117-123

2 Pellock. JM. Understanding co-morbidities affecting children with epilepsy. Neurology 2004;62:S17-S23

3 Stevanovic D, Tadic I, Novakovic T. Health-related quality of life in children and adolescents with epilepsy: a systematic review. In: Gadze PZ, ed. Epilepsy in Children-Clinical and Social Aspects. Croatia, Europe: InTech; 2011:161-186

4 Li Y, Ji CY, Qin J, Zhang ZX. Parental anxiety and quality of life of epileptic children. Biomed Environ Sci 2008;21(3):228-232

5 Miller V, Palermo TM, Grewe SD. Quality of life in pediatric epilepsy: demographic and disease-related predictors and comparison with healthy controls. Epilepsy Behav 2003;4(1):36-42

6 Turky A, Beavis JM, Thapar AK, Kerr MP. Psychopathology in children and adolescents with epilepsy: an investigation of predictive variables. Epilepsy Behav 2008;12(1):136-144 
7 Stevanovic D, Jancic J, Lakic A. The impact of depression and anxiety disorder symptoms on the health-related quality of life of children and adolescents with epilepsy. Epilepsia 2011;52(8):e75-e78

8 Wu DY, Ding D, Wang Y, Hong Z. Quality of life and related factors in Chinese adolescents with active epilepsy. Epilepsy Res 2010;90(1-2):16-20

9 Barbosa FD, Guerreiro MM, de Souza EA. The Brazilian version of the Quality of Life in Epilepsy Inventory for Adolescents: translation, validity, and reliability. Epilepsy Behav 2008;13(1):218-222

10 Benavente-Aguilar I, Morales-Blánquez C, Rubio EA, Rey JM. Quality of life of adolescents suffering from epilepsy living in the community. J Paediatr Child Health 2004;40(3):110-113

11 Devinsky O, Westbrook L, Cramer J, Glassman M, Perrine K, Camfield C. Risk factors for poor health-related quality of life in adolescents with epilepsy. Epilepsia 1999;40(12):1715-1720

12 Modi AC, Ingerski LM, Rausch JR, Glauser TA. Treatment factors affecting longitudinal quality of life in new onset pediatric epilepsy. J Pediatr Psychol 2011;36(4):466-475

13 Adewuya AO. Parental psychopathology and self-rated quality of life in adolescents with epilepsy in Nigeria. Dev Med Child Neurol 2006;48(7):600-603

14 Ronen GM, Streiner DL, Verhey LH, et al; North American Pediatric Epilepsy QOL Research Group. Disease characteristics and psychosocial factors: explaining the expression of quality of life in childhood epilepsy. Epilepsy Behav 2010; 18(1-2):88-93

15 Varni JW, Seid M, Kurtin PS. PedsQL 4.0: reliability and validity of the Pediatric Quality of Life Inventory version 4.0 generic core scales in healthy and patient populations. Med Care 2001;39(8):800-812

16 Morita DA, Glauser TA, Modi AC. Development and validation of the Pediatric Epilepsy Side Effects Questionnaire. Neurology 2012;79(12):1252-1258
17 Jovanovic M, Jocic-Jakubi B, Stevanovic D. Adverse effects of antiepileptic drugs and quality of life in pediatric epilepsy. Neurol India 2015;63(3):353-359

18 Modi AC, Junger KF, Mara CA, et al. Validation of the PedsQL Epilepsy Module: A pediatric epilepsy-specific health-related quality of life measure. Epilepsia 2017;58(11):1920-1930

19 Nadkarni J, Jain A, Dwivedi R. Quality of life in children with epilepsy. Ann Indian Acad Neurol 2011;14(4):279-282

20 Malhi P, Singhi P. Correlates of quality of life with epilepsy. Indian J Pediatr 2005;72(2):131-135

21 George J, Kulkarni C, Sarma GR. Anti-epileptic drugs and quality of life in patients with epilepsy: a tertiary care hospitalbased study. Value Health Reg Issues 2015;6:1-6

22 Sinha A, Sanyal D, Mallik S, Sengupta P, Dasgupta S. Factors associated with quality of life of patients with epilepsy attending a tertiary care hospital in Kolkata, India. Neurol Asia 2011;16(1):33-37

23 Goodwin SW, Wilk P, Karen Campbell M, Speechley KN. Emotional well-being in children with epilepsy: family factors as mediators and moderators. Epilepsia 2017;58(11):1912-1919

24 Aggarwal A, Datta V, Thakur LC. Quality of life in children with epilepsy. Indian Pediatr 2011;48(11):893-896

25 Fong CY, Chang WM, Kong AN, Rithauddin AM, Khoo TB, Ong LC. Quality of life in Malaysian children with epilepsy. Epilepsy Behav 2018;80:15-20

26 Guerrini R, Zaccara G, la Marca G, Rosati A. Safety and tolerability of antiepileptic drug treatment in children with epilepsy. Drug Saf 2012;35(7):519-533

27 Krishnamoorthy ES, Trimble MR, Blumer D. The classification of neuropsychiatric disorders in epilepsy: a proposal by the ILAE Commission on Psychobiology of Epilepsy. Epilepsy Behav 2007;10(3):349-353 